\title{
Self-Adaptive Partial Discharge Signal De-noising Based on Ensemble Empirical Mode Decomposition and Automatic Morphological Thresholding
}

\author{
Jeffery C. Chan, Hui Ma, Tapan K. Saha and Chandima Ekanayake \\ School of Information Technology and Electrical Engineering \\ The University of Queensland, Australia
}

\begin{abstract}
This paper proposes a self-adaptive technique for partial discharge (PD) signal denoising with automatic threshold determination based on ensemble empirical mode decomposition (EEMD) and mathematical morphology. By introducing extra noise in the decomposition process, EEMD can effectively separate the original signal into different intrinsic mode functions (IMFs) with distinctive frequency scales. Through the kurtosis-based selection criterion, the IMFs embedded with PD impulses can be extracted for reconstruction. On the basis of mathematical morphology, an automatic morphological thresholding (AMT) technique is developed to form upper and lower thresholds for automatically eliminating the residual noise while maintaining the PD signals. The results on both simulated and real PD signals show that the above PD denoising technique is superior to wavelet transform (WT) and conventional EMD-based PD de-noising techniques.
\end{abstract}

Index Terms - De-noising, ensemble empirical mode decomposition (EEMD), high voltage (HV) equipment, mathematical morphology, partial discharge (PD), wavelet transform (WT).

\section{INTRODUCTION}

PARTIAL discharge (PD) measurement provides an effective and convenient means for on-line monitoring of insulation systems of high voltage (HV) equipment. However, environmental interference and noise may jeopardize PD measurement and cause difficulties in PD data analysis. Therefore, necessary denosing techniques need to be implemented to extract PD signals from original noise-corrupted signals. Over the past several decades, a variety of PD signal de-noising techniques have been proposed in the literature [1,2]. Recently, a number of researchers [3-10] applied wavelet transform (WT) to PD denoising. Through the signal decomposition by WT, noise and real PD signals can be separated into different coefficients. By applying thresholds to the coefficients, noise is discarded and the PD signals are kept for reconstructing the de-noised PD signals. However, due to the needs of manually determining mother wavelets and decomposition levels, WT is not a self-adaptive and automatic decomposition technique.

To provide a fully automatic decomposition technique, especially for non-stationary and nonlinear signals, empirical mode decomposition (EMD) has been proposed [11]. EMD does not require the pre-selection of mother wavelets. Instead, it decomposes the original signal into a number of monocomponent signals called intrinsic mode functions (IMFs) by using the local characteristic time scale of the signal itself.
Recently, Tang et al applied EMD to PD de-nosing [12]. However, the inherent limitations of EMD such as mode mixing and end effect were not considered in [12]. Due to these two limitations, an IMF may consist of more than one frequency component combining both real signals and noise. Also, redundant IMFs can be generated. If these IMFs are selected for reconstruction, the de-noised PD signals can be distorted.

To address the above issues in applying WT and EMD on PD de-noising, this paper proposes a hybrid method, which adopts ensemble EMD (EEMD) for signal decomposition and utilizes mathematical morphology for automatic threshold determination. The key aspects of formulation, implementation, and verification of the hybrid method will be presented in the remainder of this paper. They are: (1) EEMD for effectively overcoming mode mixing in EMD by decomposing original noisecorrupted PD signals into IMFs for separating real PD signals from noise; (2) kurtosis-based selection method for extracting IMFs with PD impulses for reconstruction to avoid end effect in EMD; (3) automatic morphological thresholding (AMT) using mathematical morphology for automatically creating thresholds to eliminate the noise with the same frequency scales as the PD impulses in the reconstruction process; and (4) evaluation measures for comparing the de-noising performances of WT, EMD, and the proposed hybrid method. Since there are no requirements of pre-selecting mother wavelets and thresholds for different types of PD signals in the proposed method, it can be 
applied to the real situations, in which the types of defects of HV insulation systems and the properties of PD signals are unknown.

\section{BRIEF REVIEW OF SIGNAL DECOMPOSITION TECHNIQUES AND MORPHOLOGICAL FILTER}

\subsection{BRIEF REVIEW OF WT}

In WT, signals are decomposed into a series of components called coefficients ranging from the highest to the lowest frequencies scales. Such decomposition is performed based on convolution between original signal and the selected mother wavelets, which shifts along the time scale using translation and scale factors. In PD de-noising, discrete wavelet transform (DWT) is widely adopted $[3,4]$. The operation of DWT can be represented by a filter band consisting of low pass filters (LPFs) and high pass filters (HPFs) with the decomposition on the low passed signals [13].

One of the major limitations of WT is mother wavelet selection. The authors of this paper addressed this limitation in [14]. Basically, better de-noising performance can be achieved if the selected mother wavelets have high correlation with real signals. However, it was found that (1) for the signals with low signal-to-noise ratios (SNRs), mother wavelets may be selected for matching the noise rather than the real signals; and (2) fixed mother wavelets cannot match all type of PD signals for different PD defects [14].

\subsection{BRIEF REVIEW OF EMD}

EMD is an adaptive decomposition technique. Without specifying any mother wavelet, EMD decomposes a signal into a series of IMFs of different frequency scales [11]. The decomposed signal can later be precisely reconstructed. EMD uses a sifting process to eliminate riding waves and smooth uneven amplitudes that might be embedded in IMFs. Therefore, an IMF can be treated as a mono-component signal, which satisfies (1) the number of extrema must either be equal to or, at most, differ by one from the number of zero crossings; and (2) the mean value of both envelopes defined by the local maxima and local minima is zero at any point in the data [15].

It is assumed $x(t)$ is a signal being extracted in the sifting process. Firstly, local extrema of $x(t)$ are defined. Then, cubic spline interpolations are performed on both maxima and minima to get the upper envelope, $x_{U}(t)$, and lower envelope, $x_{L}(t)$. The mean of the above two envelopes is denoted as $m(t)=\left(x_{U}(t)+x_{L}(t)\right) / 2$. The iteration of deciding IMF is $I_{n i}(t)=x(t)-m_{n i}(t)$, where $I_{n i}(t)$ is the $n$-th IMF at the $i$-th iteration. $I_{n i}(t)$ is the first IMF if it satisfies the conditions of mono-component. Otherwise, the iteration will be continued on $I_{n i}(t)$. After extracting the first IMF, which embraces the highest frequency component in the original signal, the iteration will be continued on the residue, $r_{n}(t)=x(t)-I_{n i}(t)$. Replacing $x(t)$ by $r_{n}(t)$ and repeating the above process until the last IMF is obtained. A precise reconstruction, $x_{R}(t)$, can be achieved by a linear combination:

$x_{R}(t)=\sum_{n=1}^{N-1} I_{n i}(t)+r_{N}(t)$

where $N$ is the total number of IMFs and $r$ is the last IMF or residue.

However, due to the problem of mode mixing in the above sifting process, an IMF often embraces signal components with dramatically disparate frequencies scales. The signal components with similar frequency scales may also reside in different IMFs [16]. Another limitation of EMD is the end effect that may generate meaningless low frequency IMFs. This is due to the excessive decomposition of EMD, in which swings that generated from both ends of signals propagate toward the whole signal span and finally corrupt the IMFs. This paper adopts EEMD to deal with the above mode mixing problem and proposes a kurtosis-based IMF selection criterion to solve the end effect problem (refer to Section 4).

\subsection{BRIEF REVIEW OF EEMD}

Through adding white noise to the original signal, EEMD forces the sifting process to make different frequency scales collating in the proper IMFs dictated by dyadic filter bank [16].

After adding white noise on the original signal, EEMD extracts IMFs as normal EMD does. Once the extraction of the first ensemble is completed, a set of IMFs with noise is generated. Then a new white noise with the same standard deviation on the amplitude as the previously added noise is injected into the original signal again and EMD process is continued on this new noisy signal. The amplitude of white noise and ensemble number are defined as:

$\ln \varepsilon+\frac{A_{n}}{2} \ln N_{e}=0$

where $A_{n}$ is the amplitude of the added white noise, $N_{e}$ is the ensemble number, and $\varepsilon$ is the final standard deviation of error. The resulting IMFs after all the ensembles are the average value of the ensembles on each IMF. Since white noise is a zero mean random signal, the average value can cancel the errors produced by the white noise.

\subsection{MATHEMATICAL MORPHOLOGY-BASED MORPHOLOGICAL FILTER}

The purpose of using mathematical morphology-based morphological filter is to extract PD impulses in the reconstructed signals from EEMD. Mathematical morphology is originally designed for image processing. Later, its application has been extended to feature extraction and machine fault diagnosis [17-20]. The theory of mathematical morphology is based on mathematical operators, which are applied between signals and structure element. Structure element is a predefined shape, such as flat and triangular, with finite length. The extraction of impulses is defined as the extraction of local morphological features through the intersection between structure element and signals.

Two basic operators in mathematical morphology are erosion 
and dilation, which can be expressed by Minkowski subtraction and addition respectively. By combining these two operators, other two operators, opening and closing, can be created. Assuming $x_{n}$ is an one-dimensional signal with discrete function over domain $X=(0,1,2, \ldots, N-1)$ and $s_{n}$ is a structure element with discrete function over domain $S=(0,1,2, \ldots, M-1)$ [18], then the four operators can be defined as [17]:

$$
\begin{aligned}
& (X \Theta S)_{n}=\min \left(X_{(n+m)}-S_{m}\right), \quad m \in 0,1,2, \ldots, M-1 \\
& (X \oplus S)_{n}=\max \left(X_{(n-m)}+S_{m}\right), \quad m \in 0,1,2, \ldots, M-1 \\
& (X \circ S)_{n}=(X \Theta S \oplus S)_{n} \\
& (X \bullet S)_{n}=(X \oplus S \Theta S)_{n}
\end{aligned}
$$

where $\Theta, \oplus$, ०, and $\bullet$ denote erosion, dilation, opening, and closing operators respectively. It can be seen from Figure 1 that erosion operator suppresses maxima and enlarges the minima, dilation operator enlarges maxima and suppresses minima, opening operator cuts the maxima and maintains the shape of minima, and closing operator maintains the shape of maxima and cuts the minima. By selecting proper operators, PD impulses can be extracted.

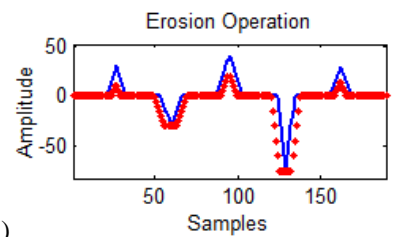

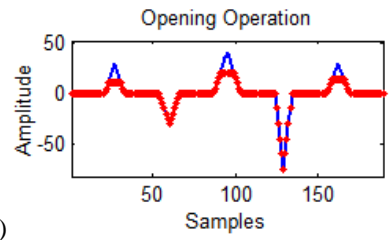

(b)
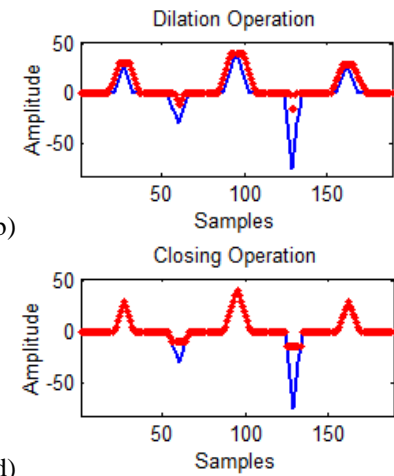

(d)

Figure 1. Results of mathematical morphology (red dots) on original signal (blue lines). (a) Erosion, (b) Dilation, (c) Opening, and (d) Closing

\section{COMPARATIVE STUDY OF DECOMPOSITION TECHNIQUES ON SIMULATED SIGNALS}

\subsection{SIMULATED PD SIGNALS}

PD signals are impulsive-type signals and their rise times can be as short as 1-2 ns at their discharge locations inside a HV apparatus [21]. However, the PD signals can be distorted and attenuated when they propagate from their respective discharge locations to PD detectors. Their rise times and durations can also be distorted. Therefore, the durations of PD signals acquired by PD detectors can be significantly different from the durations of PD signals at their occurrence locations. Chen and Czaszejko investigated influence of PD detection circuits with different circuit parameters on the characteristics of acquired PD signals [22]. Their results prove that PD impulses with oscillatory frequencies ranging from several Megahertz to several tens of Megahertz can be detected. To evaluate de-noising performances of different decomposition techniques, two types of simulated PD signals which are damped exponential pulse (DEP) and damped oscillatory pulse (DOP) were adopted. The duration of DEP and DOP is around $0.1 \mu \mathrm{s}$ and the oscillatory frequency is around $14 \mathrm{MHz}$. DEP and DOP are defined as [5]:

$$
\begin{aligned}
& D E P(t)=A\left(e^{-t / t 1}-e^{-t / t 2}\right) \\
& D O P(t)=A * \sin \left(2 \pi f_{c} t\right)\left(e^{-t / t 1}-e^{-t / t 2}\right)
\end{aligned}
$$

where $A$ is the signal amplitude, $f_{c}$ is the oscillatory frequency of DOP, and $t$ is the time constant of damping coefficients for controlling PD parameters such as rise time, pulse width, and decay time. Figure 2 shows these two types of PD signals.

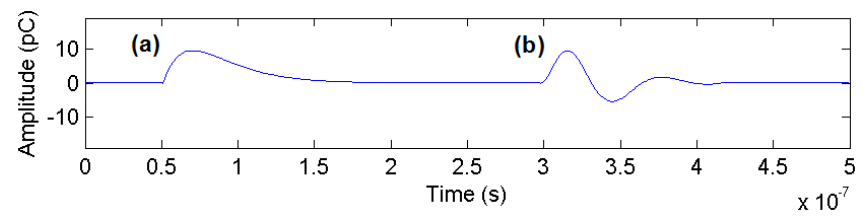

Figure 2. Simulated PD signals (a) DEP and (b) DOP

\subsection{COMPARISON OF EEMD AND DWT}

To compare EEMD with DWT, five simulated PD impulses with different amplitudes and SNR $=-1 \mathrm{~dB}$ were employed. SNR is calculated by using amplitudes of a signal. Assuming Sig be an original PD signal and Noi be the noise. SNR is defined as $10 \log _{10}\left(\sum_{i=1}^{L} \operatorname{Sig}(i)^{2} / \sum_{i=1}^{L} \operatorname{Noi}(i)^{2}\right)$, where $L$ is length (i.e. number of samples) of PD signal and noise. Figure 3 shows decomposition results of EEMD on DOP and DEPtype PD signals. The decomposition was constructed with 300 ensembles and the amplitudes of the injected white noise were 0.2 standard deviation of original signals. Figure 4 shows approximation coefficients generated from five-level decomposition of DWT. Three commonly used mother wavelets, i.e. $\mathrm{db} 2, \mathrm{db} 5$, and bior1.5, were adopted for comparison [4-6].

It can be seen from Figure 3 that noise (IMFs 1-3 in DOP and IMFs 1-4 in DEP) and PD impulses (IMFs 4-5 in DOP and IMFs 5-6 in DEP) can be separated distinctively. However, decomposition performances in DWT (Figure 4) highly depend on the mother wavelets. Choosing inappropriate mother wavelets in DWT may incur distortion of de-noised signals. In Figure 4, db5 attained better de-nosing performance compared with $\mathrm{db} 2$ and bior1.5. This is due to similarity between the approximation coefficients generated in the decomposition process using db5 and the original signals.

\subsection{COMPARISON OF EEMD AND EMD}

EMD decompositions on the above simulated signals are shown in Figure 5. It can be seen that some PD impulses are merged with noise as indicated by the red arrows in IMF 3 for DOP and IMF 4 for DEP. If these IMFs are discarded before signal reconstruction, the de-noised signals will be distorted. On the other hand, preserving these IMFs will introduce significant noise in the de-noised signals.

If thresholds are applied to the IMFs, it might be possible to filter the noise. However, if the amplitude of a PD impulse is 
similar to that of the noise in an IMF, this PD impulse may also be treated as noise and discarded. Moreover, thresholds are the same values at both positive and negative sides. Thus it is not feasible to apply the thresholds to PD signals, which consists of positive and negative impulses of different amplitudes. Therefore, this paper proposes a new approach for thresholding using mathematical morphology in PD signal denoising.

(a)

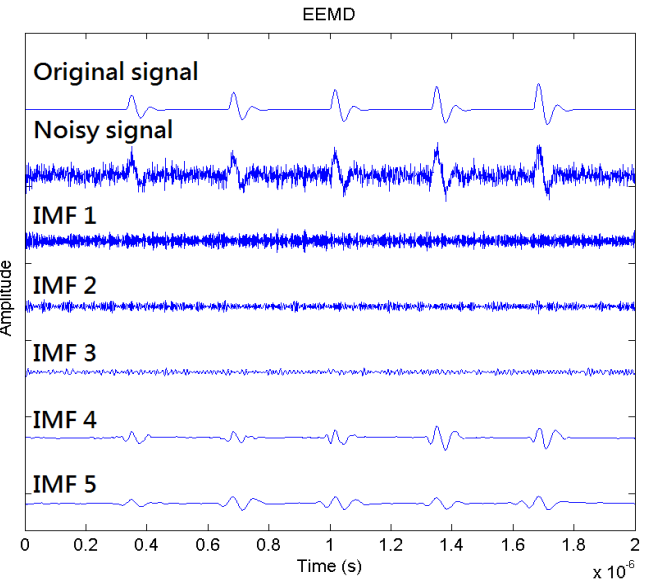

EEMD

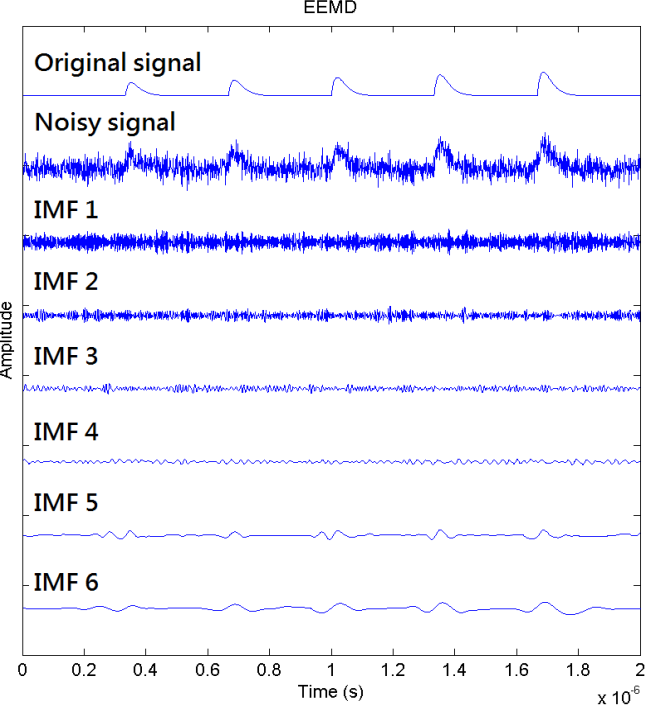

Figure 3. Decompositions of EEMD on simulated signals. (a) DOP (5 levels) and (b) DEP (6 levels)

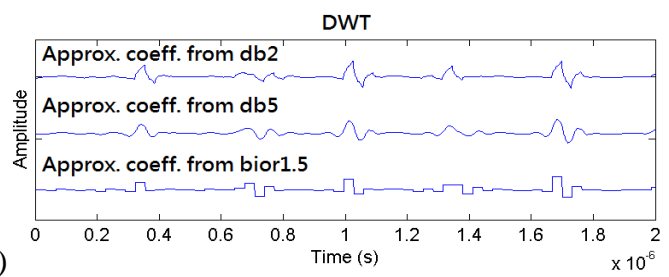

(a)

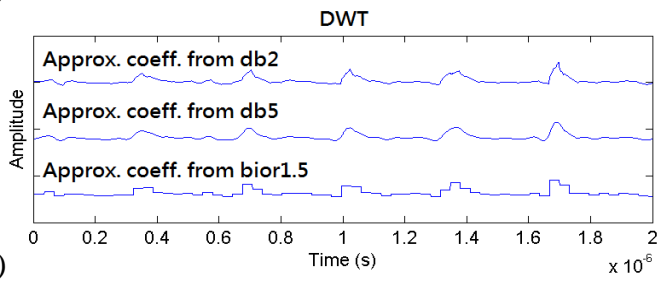

(b)

Figure 4. Approximation coefficients of DWT on simulated signals. (a) DOP and (b) DEP
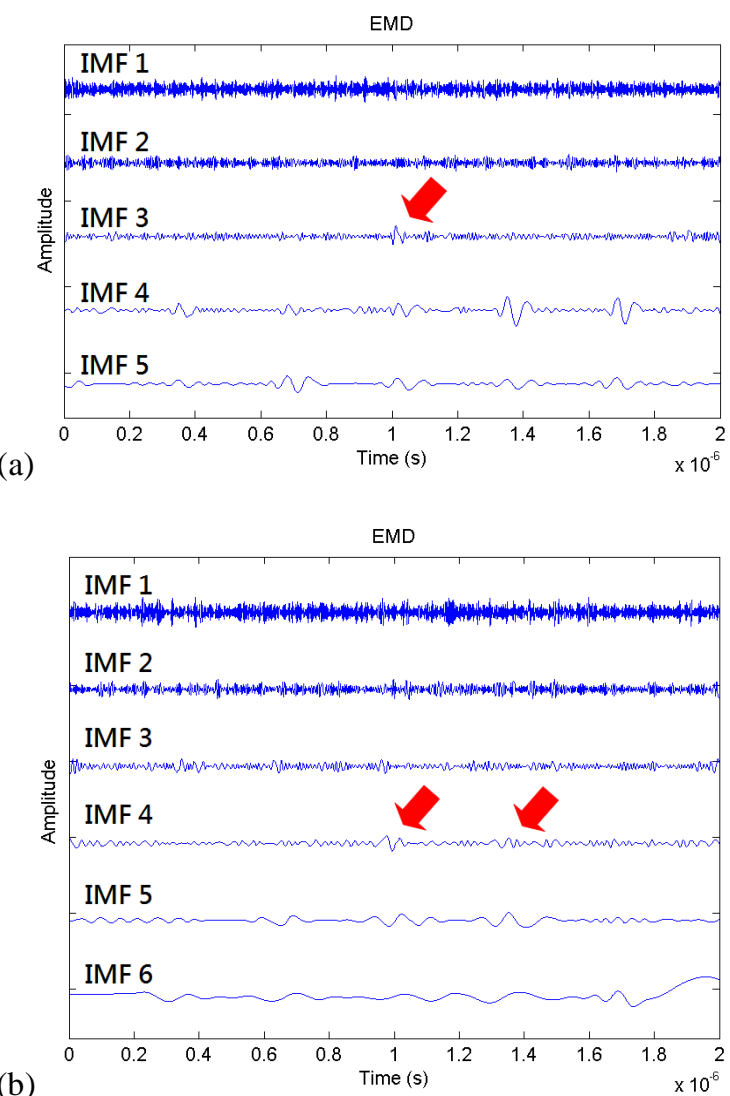

Figure 5. Decompositions of EMD on simulated signals. (a) DOP (5 levels) and (b) DEP (6 levels)

\section{A PD SIGNAL DE-NOISING METHOD BASED ON EEMD WITH AUTOMATIC THRESHOLDING}

This section presents a PD signal de-noising method, which adopts EEMD for signal decomposition and mathematical morphology-based morphological filter for automatic thresholding in signal reconstruction.

\subsection{AUTOMATIC THRESHOLDING USING MORPHOLOGICAL FILTER}

Since amplitudes of positive and negative PD impulses may not be the same, the mathematical morphology-based morphological filter calculates the threshold values separately in both positive and negative sides of PD signals. The major difficulties of applying mathematical morphology are the selections of shape and length of structure element. Inappropriate selection of these two parameters can lead to excessive suppression of extrema and consequently the distorted signals.

A number of structure element selection schemes have been proposed in the literature [17-20]. In [17, 20], flat structure element was adopted for extracting impulsive periodic signals. The lengths of structure element were decided based on the repetition periods. However, this approach requires prior knowledge of the repetitive frequencies of the signals, which might not be readily available in some applications. In [19], 
sinusoidal structure element was used and the lengths were selected based on decay rate of the structure element for periodic bearing signals. However, it is not a trivial task to decide the decay coefficient, natural frequency, and the amplitude of the structure element for such periodic signals. In [18], triangular structure element was applied to impulsive periodic signals. The limitation of this type of structure element is that it may not match all types of impulses when the amplitudes need to be considered.

In this paper, flat structure element is employed due to the simplicity in calculation and the shape has little effect on the analysis [20]. To illustrate the selection of length of structure element, a simulated signal with different sampling points ranging from 2 to 7 apart between the impulses was generated (Figure 6). Closing operator was used for extracting positive peaks of the signal as denoted by the red dots. It can be seen that the length of structure element should be shorter than or equal to the intervals of impulses for effective signal extraction.

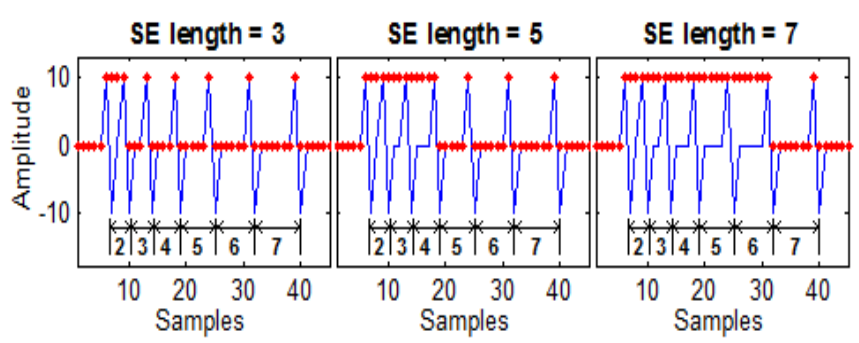

Figure 6. Results of closing operator (red dots) with different lengths of SEs

To automatically define the lengths of structure element for creating thresholds, this paper develops an AMT technique as shown in Figure 7. AMT starts with determination of sample number, $\mathrm{S}_{\mathrm{AC}}$, in each $\mathrm{AC}$ cycle and window number, $\mathrm{N}_{\mathrm{w}}$. Maximum 32 windows are used to provide a desirable resolution while maintaining reasonable computational time. The lengths of structure elements can be set from $S_{A C} / N_{w}$ to $S_{A C}$. Then, closing and opening operations adopting these structure elements are performed to create upper and lower envelopes respectively. Energy is defined as sum of the square of amplitude for each data point in each envelope. With the increment of structure element length, more peaks are enclosed and gradually the energy values of the envelopes will increase. However, for the envelopes covering the peaks with small intervals or the peaks with large intervals but small amplitudes, the increase of energy will not be too much (middle part of Figures $8 \mathrm{a}$ and $8 \mathrm{~b}$ ). In contrast, if the envelopes cover the peaks with large intervals and amplitudes, energy values will increase dramatically (right-hand side of Figures $8 \mathrm{a}$ and $8 \mathrm{~b}$ ).

Taking into account the above scenarios, optimal thresholds are determined in two steps: (1) taking average on upper/lower envelopes that provide the longest successive similar energy values ( $\pm 10 \%$ of energy values); and (2) obtaining the optimal thresholds by taking the mean values of the averaged envelopes (Figure 8c).

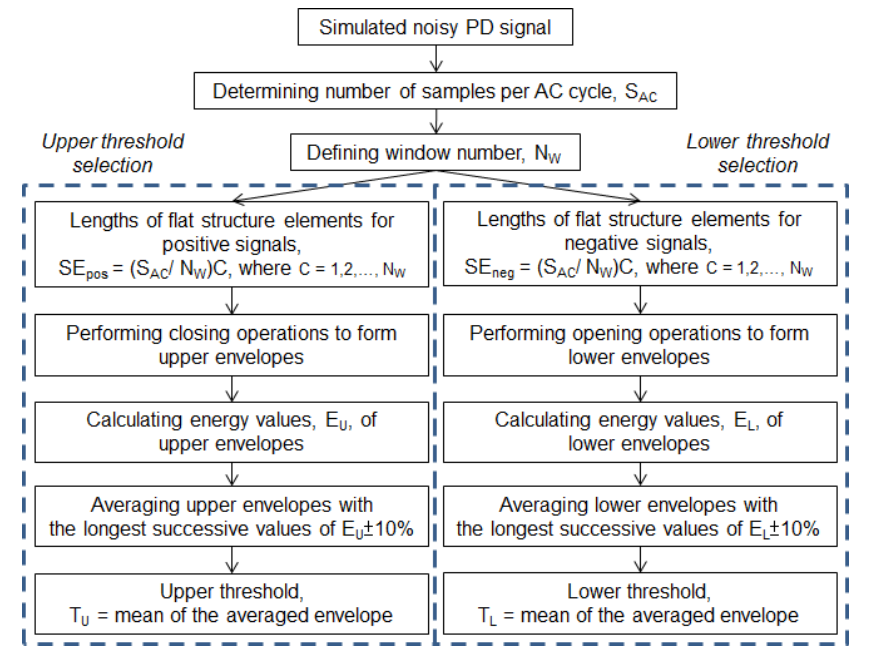

Figure 7. Flowchart of AMT

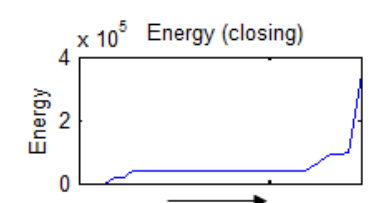

(a) Increasing length of structure element

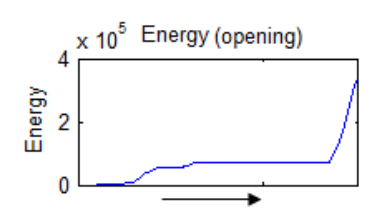

(b) Increasing length of structure element (c)

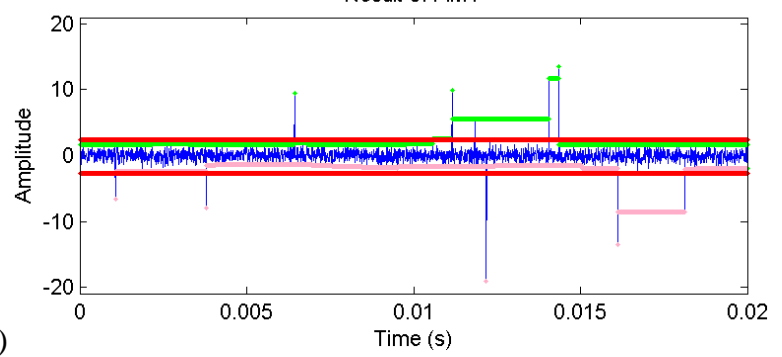

Figure 8. Results of AMT on a noisy DEP-type signal. (a) Energy values of upper envelops, (b) Energy values of lower envelopes, and (c) Averaged upper (green dots) and lower envelopes (pink dots), and thresholds (red lines)

\subsection{PROPOSED SELF-ADAPTIVE PD SIGNAL DE-NOISING METHOD}

The overall flowchart of the proposed self-adaptive method for PD signal de-noising is depicted in Figure 9. White noise and periodic noise which are very common in PD data acquisition [3-5] were injected to the original PD signal. During on-line PD measurement, white noise can be generated from ambience, whereas periodic noise can be generated from communication systems, radio transmissions, and power cycles [3-5]. In this paper, the injected periodic noise consists of three sinusoidal signals with randomly selected amplitudes and frequencies. The largest amplitude of these signals can be up to the absolute value of the largest amplitude of an original acquired PD signal. The frequencies of these signals are with the range up to $1 \mathrm{kHz}$ to simulate signal fluctuations that could be appeared in on-line PD measurements.

Firstly, EEMD is executed to decompose the noisy signals. The ensemble number is set to 300 and the amplitude of the injected white noise is 0.2 standard deviation of the original signal. According to [23], an IMF generally satisfies the 
Cauchy condition after five iterations in the sifting process. More iterations could not change IMFs significantly. Thus ten iterations are used to guarantee the stability and convergence of IMFs. After EEMD decomposition, kurtosis is applied to select the IMFs with PD impulses due to the sensitivity of transient signals. In other words, larger value of kurtosis refers to a signal consisting of abruptly changed impulses while smaller value of kurtosis refers to a slowly fluctuated signal or a signal consisting of evenly distributed amplitudes (e.g. white noise and periodic signal). The kurtosis, $k$, is defined as:

$k=\frac{\sum_{i=1}^{L}\left(I_{i}-\bar{I}\right)^{4}}{(L-1) \sigma^{4}}$

where $\bar{I}, L$, and $\sigma$ are the mean, length, and standard deviation of IMF respectively.

The selection process starts from IMF1 which comprises the highest frequency component of the originally noise-corrupted signal. If the kurtosis of an IMF is suddenly dropped to half of that of previous IMF, it implies that the IMF with the lower kurtosis value consists of fluctuations in low frequency, periodic noise, and/or white noise. Therefore, it is considered as pure noise without containing any PD impulses and discarded. Otherwise, it is added to the previous IMF for reconstruction and the selection will be continued on the remaining IMFs. Such kurtosis-based selection can pick up the noisy IMFs and eliminate the low frequency IMFs that are generated from end effect.

After the above selection process, reconstruction can be created. Since some noise with the same frequency scales of the selected IMFs may be included in the reconstructed signal, the AMT can be employed to create upper and lower thresholds of the signal. De-noised signal can then be produced by applying hard thresholding.

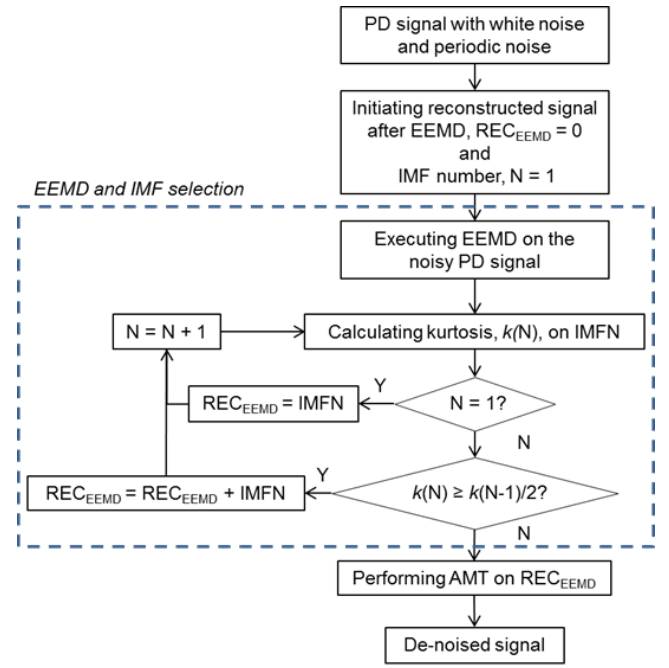

Figure 9. The proposed self-adaptive PD signal de-noising method

\section{EXPERIMENTAL SETUP}

PD signals were acquired by a commercially available equipment (Omicron, MPD 600), which complies with IEC60270 standard [24] as shown in Figure 10.

Five different PD models (Figure 11) were constructed to simulate different insulation defects in HV equipment. Model 1 simulates internal discharge. It consists of three pressboards with a void at the center of the middle pressboard between the $\mathrm{HV}$ and grounding electrodes. Model 2 simulates floating discharge by putting four metal particles on a pressboard between $\mathrm{HV}$ and grounding electrodes. Model 3 simulates a protrusion of conductor by using needle-sphere configuration. Model 4 simulates normal insulation condition with 1 pressboard between $\mathrm{HV}$ and grounding electrodes. Model 5 simulates corona using needle-plane configuration. Models 1 to 4 were immersed in mineral oil, whereas model 5 was in air.

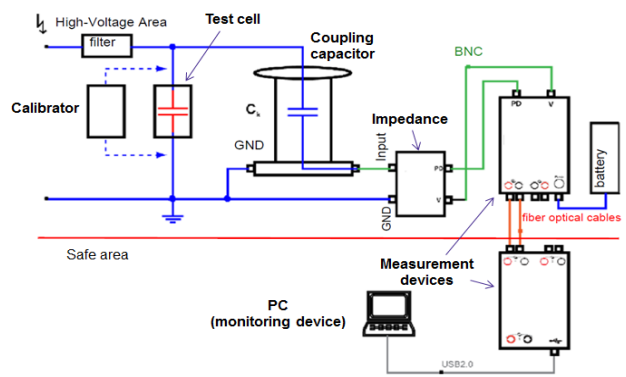

Figure 10. Experimental setup [24]

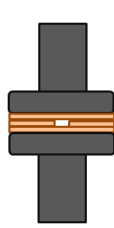

(a)

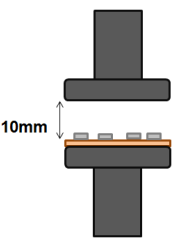

(b)

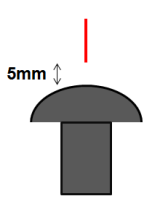

(c)

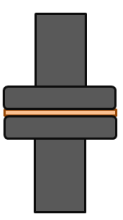

(d)

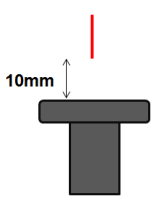

(e)
Figure 11. PD source models. (a) Model 1 - pressboards with 1 void (in oil), (b) Model 2 - pressboard with 4 metal particles (in oil), (c) Model 3 - needle sphere (in oil), (d) Model 4 - 1 pressboard (in oil), and (e) Model 5 - needle plane (in air)

\section{RESULTS AND ANALYSIS}

\subsection{EVALUATION MEASURES}

To evaluate the de-noising performance of the proposed method, three measures including mean square error (MSE), $\mathrm{M}$, correlation coefficient (CC), $\gamma$, and impulse number error, $I E$, were adopted. They are defined as:

$$
\begin{aligned}
& \mathrm{M}=\frac{1}{L} \sum_{i=1}^{L}\left(O_{i}-D_{i}\right)^{2} \\
& \gamma=\frac{\sum_{i=1}^{L}\left(O_{i}-\bar{O}\right)\left(D_{i}-\bar{D}\right)}{\sqrt{\sum_{i=1}^{L}\left(O_{i}-\bar{O}\right)^{2} \sum_{i=1}^{L}\left(D_{i}-\bar{D}\right)^{2}}} \\
& I E=\left|I M_{o}-I M_{d}\right|
\end{aligned}
$$

where $L$ is the length of signal, $O$ denotes the original signal, $D$ denotes the de-noised signal, $\bar{O}$ and $\bar{D}$ are the mean values of $O$ and $D$ respectively, $I M_{o}$ is the number of impulse in original signal, and $I M_{d}$ is the number of impulse in de-noised signal.

Since different types of insulation defects produce different patterns of PD signals, $\mathrm{M}$ and $\gamma$ can be used to indicate the 
similarity of $\mathrm{PD}$ patterns whereas $I E$ is for examining the integrity of PD impulses.

\subsection{RESULTS ON SIMULATED PD SIGNALS}

This section provides comparison between the proposed method, DWT and EMD-based methods for PD signal denoising on the simulated signals. In the comparison, DWTbased method applied a commonly used threshold to the coefficients with five decomposition levels [5]. EMD-based method used the same approach of the proposed method but the decomposition of EEMD was replaced by EMD. It is termed as EMD+AMT in the remaining of the paper. The results and evaluations of DWT and EMD+AMT are shown in Figure 12 and Table 1.

Figure 12 presents de-noised DEP-type PD signals ( SNR = $5 \mathrm{~dB}$ ) obtained from DWT (mother wavelet $=\mathrm{db} 5$ ), EMD+AMT, and the proposed method. It can be observed that some impulses are missing in the de-noised signals obtained by DWT (right-hand side of Figure 12c). This could be caused by excessive noise rejection when thresholds were applied to the coefficients. Also, the impulses' polarities (positive and negative directions) are ambiguous. Although the polarities of impulses can be preserved by using EMD+AMT, noise still exists while some impulses disappear in the de-noised signal (right-hand side of Figure 12d). Such remaining noise comes from mode mixing of EMD, in which the selected IMFs embrace relatively low frequency and large fluctuation periodic noise as well as PD impulses. Therefore, the periodic noise still appears in the reconstructed signal of EMD as shown in the left-hand side of Figure 12d. Since AMT is originally designed for filtering noise in the reconstructed signals of EEMD (left-hand side of Figure 12e) without the present of mode mixing, some noise can thus still be found in the de-noised signal of EMD+AMT.

It can be clearly observed that the proposed method outperforms DWT and EMD+AMT. The de-noised signal preserves all the PD impulses with correct polarities and locations and only has minor changes in the amplitudes (righthand side of Figure 12e).

Table 1 tabulates the results in terms of the three evaluation measures with varying SNRs. In the table, " $\downarrow \mathrm{M}(\%) ", " \uparrow \gamma(\%) "$, and " $\downarrow I E(\%) "$ refer to the percentage of MSE reduction, CC increment, and impulse number error reduction respectively of the proposed method with respect to the corresponding denoising methods (i.e. DWT and EMD+AMT). The results of DWT in Table 1 are the average of the results obtained from various mother wavelets including $\mathrm{db} 2, \mathrm{db} 4, \mathrm{db} 5, \mathrm{db} 10, \mathrm{db} 25$, $\mathrm{db} 45$, bior1.5, bior6.8, and coif5.

It can be observed from Table 1 that the amount of MSE reductions and $\mathrm{CC}$ increments are larger when SNRs are smaller in both the comparisons of DWT and EMD+AMT for DOP (Table 1a) and DEP (Table 1b). The amount tends to reduce when the SNRs become larger. This indicates that the de-nosing performance of the proposed method is close to that of DWT and EMD+AMT in slight noise situation. On the other hand, this implies that the proposed method is capable of extracting PD signals contaminated by severe noise.
When compared with the performances in terms of impulse number error, larger reduction can be found in the comparisons of DWT in both DOP and DEP. This complies with the left-hand side of Figure 12c, which reveals that the de-noised signals obtained by DWT consist of many small impulses. In Table $1,100 \%$ of $I E$ indicates that there is no addition/deduction of impulse in the proposed method, whereas "N/A" means that no addition/deduction of impulse in both the proposed and the compared methods. It can be seen that there is no addition/deduction of impulse produced in the de-noised signals obtained by the proposed method with SNRs from $1 \mathrm{~dB}$ to $5 \mathrm{~dB}$.

(a)
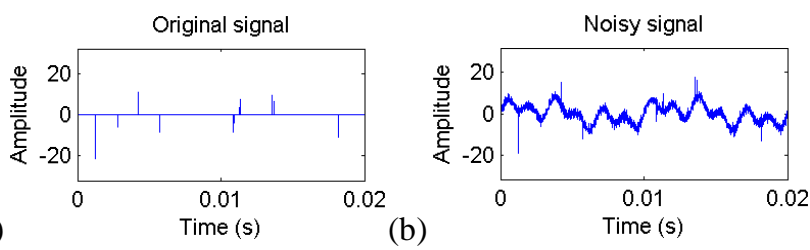

(b)

De-noised signal

(c)
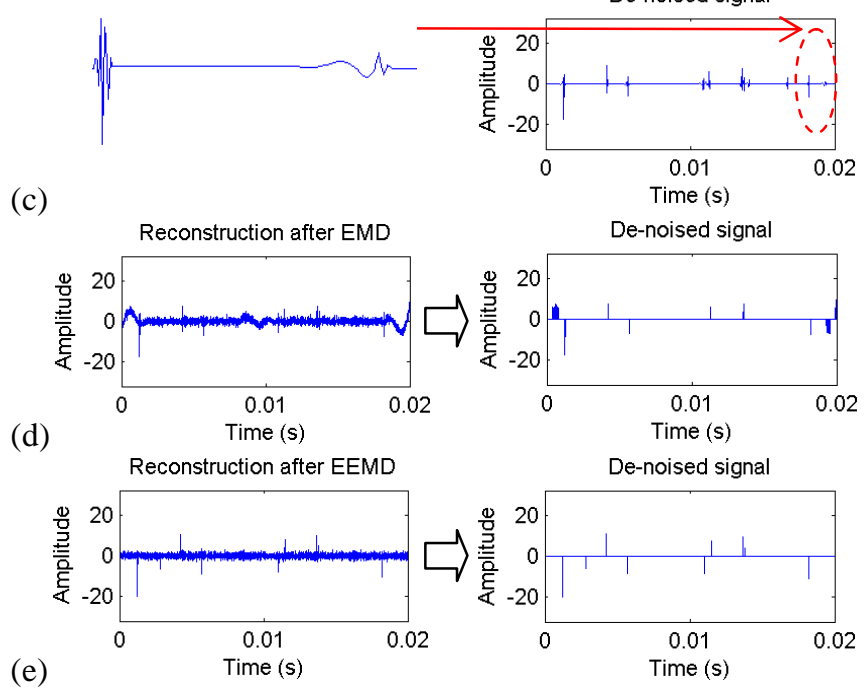

Figure 12. De-noising results of a DEP-type signal. (a) Original signal, (b) Noisy signal (SNR=-5dB), (c) Impulses enlargement and de-noised signal of DWT (db5), (d) Reconstructed and de-noised signals of EMD+AMT, and (e) Reconstructed and de-noised signals of proposed method

Table 1. Comparisons of measures on (a) DOP-type and (b) DEP-type signals

\begin{tabular}{|c|c|c|c|c|c|c|}
\hline \multirow{3}{*}{$\begin{array}{l}\text { (a) } \\
\text { SNR } \\
\text { (dB) }\end{array}$} & \multicolumn{6}{|c|}{ DOP } \\
\hline & \multicolumn{3}{|c|}{ Comparison with DWT } & \multicolumn{3}{|c|}{ Comparison with EMD+AMT } \\
\hline & $\downarrow \mathrm{M}(\%)$ & $\uparrow \gamma(\%)$ & $\downarrow I E(\%)$ & $\downarrow \mathrm{M}(\%)$ & $\uparrow \gamma(\%)$ & $\downarrow I E(\%)$ \\
\hline 5 & 25 & 4 & 100 & 45 & 14 & N/A \\
\hline 3 & 34 & 6 & 100 & 59 & 17 & 100 \\
\hline 1 & 32 & 7 & 100 & 76 & 31 & 100 \\
\hline-1 & 55 & 12 & 97 & 87 & 48 & 85 \\
\hline-3 & 60 & 25 & 99 & 87 & 46 & 76 \\
\hline-5 & 69 & 34 & 99 & 96 & 52 & 74 \\
\hline
\end{tabular}

\begin{tabular}{ccccccc}
\hline (b) & \multicolumn{6}{c}{ DEP } \\
\cline { 2 - 7 } SNR & \multicolumn{7}{c}{ Comparison with DWT } & \multicolumn{3}{c}{ Comparison with EMD+AMT } \\
(dB) & $\downarrow \mathrm{M}(\%)$ & $\uparrow \gamma(\%)$ & $\downarrow I E(\%)$ & $\downarrow \mathrm{M}(\%)$ & $\uparrow \gamma(\%)$ & $\downarrow I E(\%)$ \\
5 & 68 & 5 & 100 & 59 & 8 & N/A \\
3 & 71 & 12 & 100 & 68 & 6 & N/A \\
1 & 71 & 15 & 100 & 65 & 13 & 100 \\
-1 & 74 & 22 & 99 & 76 & 17 & 89 \\
-3 & 85 & 28 & 96 & 79 & 24 & 87 \\
-5 & 87 & 39 & 97 & 91 & 46 & 72 \\
\hline
\end{tabular}




\subsection{RESULTS ON REAL PD SIGNALS}

PD signals acquired from the five PD models (Figure 11) with added noise were used to compare the de-noising performances of DWT, EMD+AMT, and the proposed method. Figure 13 presents both time and phase-resolved diagrams of the original signal, noisy signal, and de-noised signals in $2 \mathrm{AC}$ cycles for model 1 . Figure 14 presents the de-nosing results of models 3 and 5 (due to the space limitation, the results of other two models are not provided here). Table 2 compares the denosing performances of DWT, EMD+AMT, and the proposed method with the signals obtained from $100 \mathrm{AC}$ cycles.

From Figures 13 and 14, it can be seen that, the polarities of most impulses after de-noising by DWT are blurred. Such situation is especially serious in model 1 (Figure 13c1): a whole cluster of PD impulses at the right-hand side shifts from positive to the middle position; and a large amount of impulses disappear. The result can also be observed in the corresponding phase diagram (Figure 13c2). It can also be seen from Figures 13 and 14 that some impulses are missing and noise still remains in the de-noised signals obtained by EMD+AMT. The results reveal the superiority of the proposed method. It can effectively remove the noise while maintaining the integrity of PD signals by preserving the real PD impulses and their locations.

(a1)

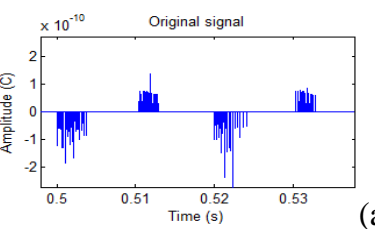

(a2)
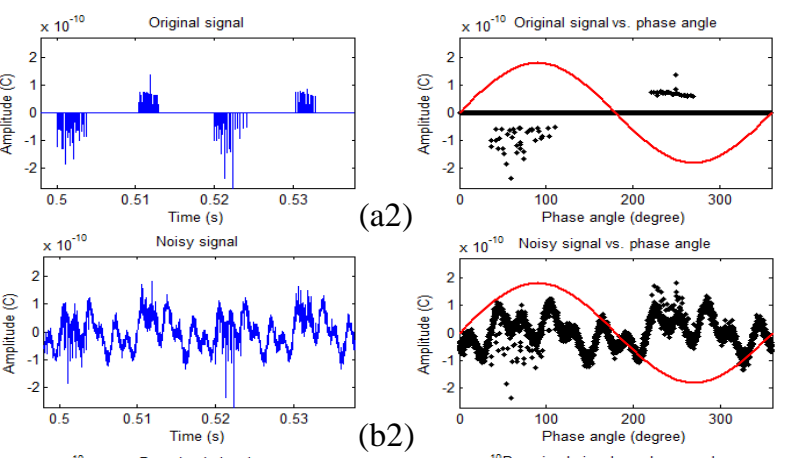

(b1)

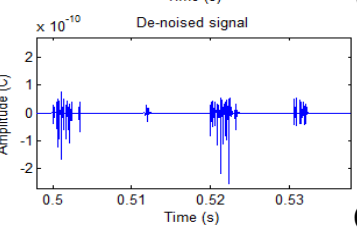

(c1)

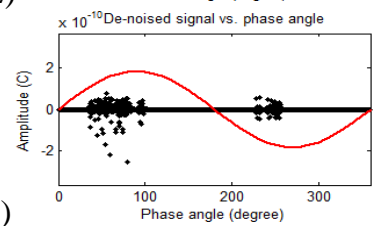

(c2)

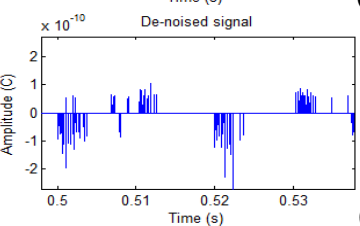

(d1)
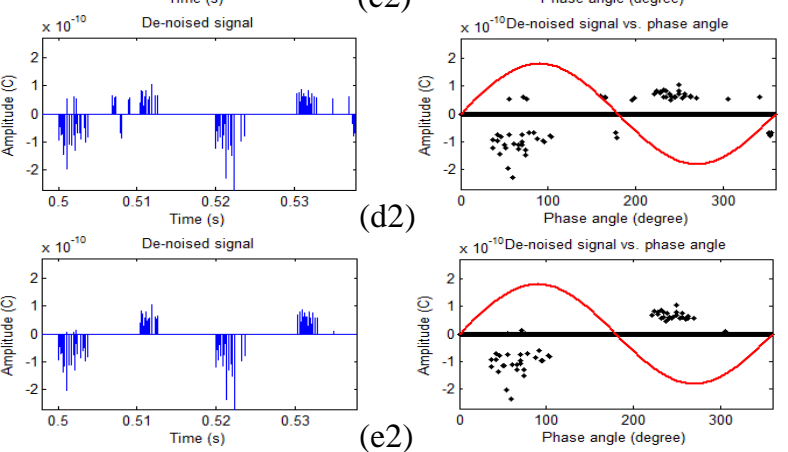

Figure 13. De-noising results (2 AC cycles) of model 1. (a1)-(e1) Original signal, noisy signal ( $\mathrm{SNR}=-5 \mathrm{~dB})$, and de-noised signals of DWT (db5), EMD+AMT, and proposed method, and (a2)-(e2) The corresponding phase diagrams
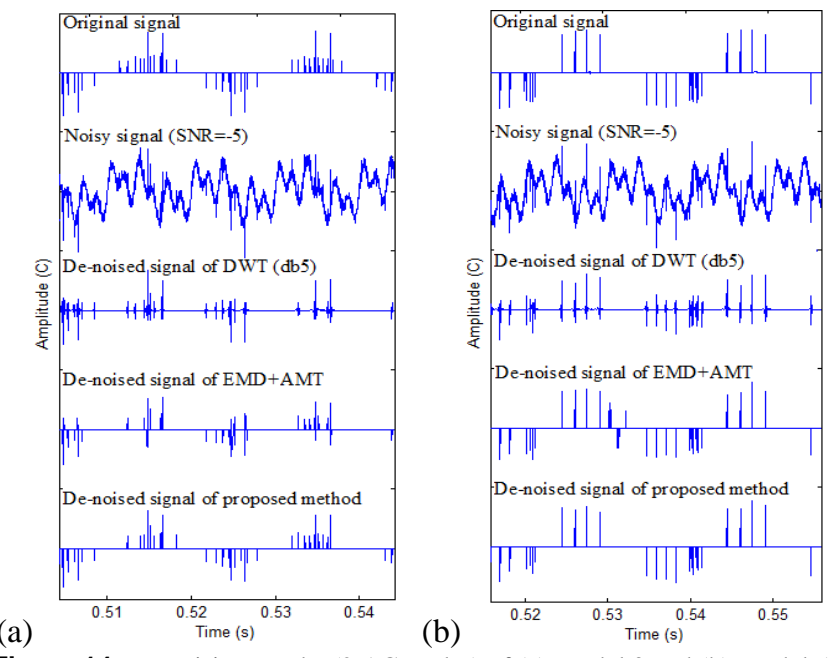

Figure 14. De-noising results (2 AC cycles) of (a) Model 3 and (b) Model 5

Table 2. Comparisons on real PD signals with $\mathrm{SNR}=-5 \mathrm{~dB}$ (100 AC cycles)

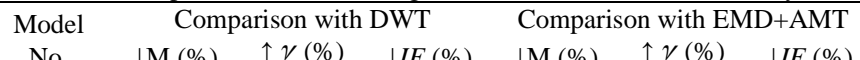

\begin{tabular}{ccccccc} 
No. & $\downarrow \mathrm{M}(\%)$ & $\uparrow \gamma(\%)$ & $\downarrow I E(\%)$ & $\downarrow \mathrm{M}(\%)$ & $\uparrow \gamma(\%)$ & $\downarrow I E(\%)$ \\
1 & 74 & 32 & 99 & 44 & 6 & 0 \\
2 & 81 & 14 & 99 & 87 & 24 & 92 \\
3 & 81 & 29 & 96 & 81 & 27 & 40 \\
4 & 68 & 12 & 97 & 28 & 15 & 0 \\
5 & 91 & 23 & 96 & 87 & 12 & 14 \\
\hline
\end{tabular}

For the results in Table 2 with 100 AC cycles of PD signals, the proposed method still outperforms the others in all five models. A higher reduction of impulse number error can be noticed in the comparison of DWT when compared with that in EMD+AMT. The results imply that the wavelet-based denoising technique may not be suitable for PD signals de-nosing since additional impulses can be induced in the de-noised signals.

\section{DISCUSSION}

This section discusses some practical issues on applying the proposed method for PD signal de-noising. The directions of improving the proposed method will also be identified.

\subsection{ROBUSTNESS OF PROPOSED METHOD ON DIFFERENT TYPES OF NOISE}

There are different types of noise in PD measurements, which include periodic noise, white noise, and stochastic transient noise. Periodic noise that consists of sinusoidal components can be generated by communication systems, radio transmissions, and power cycles. The frequency range of this type of noise can be up to $2000 \mathrm{MHz}$ [3-5]. White noise is a wide-band noise, which can be generated from ambiance and its frequency components distribute over entire spectrum. Stochastic transient noise is also a kind of wide-band noise, which can be generated by automobile spark plugs and detection circuits [5].

The method proposed in this paper aims to remove lower frequency periodic noise (up to $1000 \mathrm{kHz}$ ) and white noise. For removing the periodic noise, notch filter can be used [2]. However, frequencies of the noise can vary depending on the noise sources, notch filter is not able to provide an adaptive 
means for de-noising. Bandwidth and forgetting factor are required to be selected a priori $[2,25]$. Although DWT and EMD can also extract and remove periodic noise based on decomposition, their decomposition abilities are limited as shown in the comparative study in Section 3 and results in Section 6. The de-noising performance of DWT is limited by the selected mother wavelets, whereas EMD is not able to clearly separate noise from PD signals.

For removing white noise, the above limitations of DWT and EMD also exist (please refer to the discussion in Section 3 and Section 6). By contrast, by using the proposed method, both periodic and white noise can be separated from PD signals adaptively without considering the mother wavelet selection. Also, due to the ability of separating signals in distinctive frequency bands, the proposed method is able to effectively extract PD signals from the noise during decomposition.

Removing stochastic noise is the most challenging task in PD de-noising due to the similarity between the characteristics of stochastic noise and PD signals. Further investigation is required for removing stochastic noise from the original acquired PD signals.

\subsection{COMPUTATIONAL TIME ON ON-LINE PD MEASUREMENT}

For on-line PD measurements, computational time is crucial for PD signal de-noising and subsequently PD sources recognition. Table 3 provides the comparisons of computational time using different de-noising methods on different numbers of power cycles. The PD signals were sampled at around $1 \mathrm{GHz}$ and then re-sampled to $1 \mathrm{MHz}$ for processing. A program was written in non-optimized MATLAB and ran on a $3.4 \mathrm{GHz}, 8 \mathrm{~GB}$ RAM desktop computer for counting the time. In Table 3 , the computational time is obtained based on an average of computational time for five de-noising processes.

Table 3. Comparisons on computational time

\begin{tabular}{cccc}
\hline \multirow{2}{*}{$\begin{array}{c}\text { No. of } \\
\text { power cycle }\end{array}$} & DWT & EMD+AMT & $\begin{array}{c}\text { Proposed } \\
\text { method }\end{array}$ \\
\cline { 2 - 4 } 10 & 0.0013 & 0.0009 & 0.2028 \\
20 & 0.0013 & 0.0037 & 0.8948 \\
30 & 0.0013 & 0.0060 & 1.4563 \\
40 & 0.0013 & 0.0083 & 2.0127 \\
50 & 0.0013 & 0.0108 & 2.6624 \\
60 & 0.0013 & 0.0131 & 3.0522 \\
70 & 0.0013 & 0.0169 & 3.7461 \\
80 & 0.0013 & 0.0184 & 4.3945 \\
90 & 0.0013 & 0.0224 & 5.3046 \\
100 & 0.0013 & 0.0313 & 7.8469 \\
\hline
\end{tabular}

From the table, it can be seen that the computational time is different between different de-noising methods. Also, longer time is required for de-noising for EMD+AMT and proposed method if the PD signals are longer. On the contrary, the computational time remains unchanged in DWT. On the whole, DWT requires the shortest time among all methods, while the proposed method needs the longest time due to the ensemble process in EEMD.
From the results, although DWT and EMD+AMT require relatively short computational time, their de-noising performances as discussed in this paper cannot be satisfied. The proposed method outperforms these two methods in PD signal de-noising. Therefore, for practical on-line PD measurements, there will be a trade-off between de-noising performance and computational time. This issue needs to be investigated in future research. The possibility of improving the processing speed of the proposed method without significantly compromising its performance also needs to be investigated.

\section{CONCLUSION}

This paper proposed a self-adaptive PD de-noising technique by using EEMD and mathematical morphology to deal with the limitations of wavelet and EMD-based denoising techniques. By using a kurtosis-based selection criterion, the proposed method can effectively extract IMFs embedded with real PD impulses for reconstruction while discard the IMFs due to noise. With the mathematical morphology-based AMT technique, upper and lower thresholds can be automatically formed in the reconstruction for further eliminating the noise with the same frequency scales of the real PD impulses. Comparative study on both simulated and real PD signals demonstrated that the proposed method can preserve the integrity of PD signals without compromising the polarities and quantity of PD impulses.

\section{ACKNOWLEDGMENT}

The authors gratefully acknowledge Australian Research Council, Powerlink Queensland, Energex, Ergon Energy, and TransGrid for providing supports for this work and the anonymous reviewers for their valuable comments and suggestions.

\section{REFERENCES}

[1] T. Abdel-Galil, A. Ei-Hag, A. Gaouda, M. Salama, and R. Bartnikas, "De-noising of partial discharge signal using eigendecomposition technique," IEEE Trans. Dielectr. Electr. Insul., vol. 15 , pp. 1657-1662, 2008.

[2] S. Sriram, S. Nitin, K. Prabhu, and M. Bastiaans, "Signal denoising techniques for partial discharge measurements," IEEE Trans. Dielectr. Electr. Insul., vol. 12, pp. 1182-1191, 2005.

[3] X. Zhou, C. Zhou, and I. Kemp, "An improved methodology for application of wavelet transform to partial discharge measurement dienoising," IEEE Trans. Dielectr. Electr. Insul., vol. 12, pp. 586594, 2005

[4] H. Zhang, T. Blackburn, B. Phung, and D. Sen, "A novel wavelet transform technique for on-line partial discharge measurements part 1: WT de-noising algorithm," IEEE Trans. Dielectr. Electr. Insul., vol. 14, pp. 3-14, 2007.

[5] X. Ma, C. Zhou, and I. Kemp, "Automated wavelet selection and thresholding for PD detection," IEEE Elect. Insul. Mag., vol. 18, no. 2, pp. 37-45, 2002.

[6] X. Song, C. Zhou, D. Hepburn, and G. Zhang, "Second generation wavelet transform for data denoising in PD measurement," IEEE Trans. Dielectr. Electr. Insul., vol. 14, pp. 1531-1537, 2007.

[7] C. Chang, J. Jin, S. Kumar, Q. Su, T. Hoshino, M. Hanai, and N. Kobayashi, "Denoising of partial discharge signals in wavelet packets domain," IEE Proc. Sci. Meas. Technol., vol. 152, pp. 129-140, 2005. 
A. Gaouda, A. Ei-Hag, T. Abdel-Galil, M. Salama, and R. Bartnikas, "On-line detection and measurement of partial discharge signals in a noisy environment," IEEE Trans. Dielectr. Electr. Insul., vol. 15, pp. 1162-1173, 2008.

J. Li, T. Jiang, S. Grzybowski, and C. Cheng, "Scale dependen wavelet selection for de-noising of partial discharge detection," IEEE Trans. Dielectr. Electr. Insul., vol. 17, pp. 1705-1714, 2010.

[10] D. Dey, B. Chatterjee, S. Chakravorti, and S. Munshi, "Crosswavelet transform as a new paradigm for feature extraction from noisy partial discharge pulses," IEEE Trans. Dielectr. Electr. Insul., vol. 17, pp. 157-166, 2010

[11] N. Huang, Z. Shen, S. Long, M. Wu, H. Shih, Q. Zheng, N. Yen, C. Tung, and H. Liu, "The empirical mode decomposition and the Hilbert spectrum for nonlinear and non-stationary time series analysis," Proc. Roy. Soc. Lond. Ser. A - Math. Phys. Eng. Sci., vol. 454, pp. 903-995, 1998.

[12] Y. Tang, C. Tai, C. Su, C. Chen, and J. Chen, "A correlated empirical mode decomposition method for partial discharge signal denoising," Meas. Sci. Technol., vol. 21, pp. 1-11, 2010.

[13] S. Mallat, "A theory for multiresolution signal decomposition: the wavelet representation," IEEE Trans. Pattern Anal. Mach. Intell., vol. 11, pp. 674-693, 1989.

[14] J. Chan, H. Ma, T. Saha, and C. Ekanayake, "A novel level-based automatic wavelet selection scheme for partial discharge measurement," Proc. Australasian Universities Power Eng. Conf., Bali, Indonesia, pp. 1-6, 2012.

[15] J. Chan and P. Tse, "A novel, fast, reliable data transmission algorithm for wireless machine health monitoring," IEEE Trans. Rel., vol. 58, pp. 295-304, 2009.

[16] Z. Wu and N. Huang, "Ensemble empirical mode decomposition: a noise-assisted data analysis method," Adv. Adapt. Data Anal., vol. 1 ,pp. 1-41, 2009.

[17] N. Nikolaou and I. Antoniadis, "Application of morphological operators as envelope extractors for impulsive-type periodic signals," Mech. Syst. Signal Process., vol. 17, pp. 1147-1162, 2003.

[18] L. Zhang, J. Xu, J. Yang, D. Yang, and D. Wang, "Multiscale morphology analysis and its application to fault diagnosis," Mech. Syst. Signal Process., vol. 22, pp. 597-610, 2008.

[19] J. Wang, G. Xu, Q. Zhang, and L. Liang, "Application of improved morphological filter to the extraction of impulsive attenuation signals," Mech. Syst. Signal Process., vol. 23, pp. 236245,2009

[20] Y. Dong, M. Liao, X. Zhang, and F. Wang, "Faults diagnosis of rolling element bearings based on modified morphological method," Mech. Syst. Signal Process., vol. 25, pp. 1276-1286, 2011.

[21] R. Bartnikas, "Partial discharges their mechanism, detection and measurement," IEEE Trans. Dielectr. Electr. Insul., vol. 9, pp. 763$808,2002$.

[22] S. Chen and T. Czaszejko, "Partial discharge test circuit as a spark-gap transmitter," IEEE Elect. Insul. Mag., vol. 27, no. 3, pp. 36-44, 2011.

[23] Z. Wu and N. Huang, "A study of the characteristics of white noise using the empirical mode decomposition method," Proc. Roy. Soc. Lond. Ser. A - Math. Phys. Eng. Sci., vol. 460, pp. $1597-$ 1611, 2004.

[24] IEC International Standard 60270, "High-voltage test techniques partial discharge measurements," International Electrotechnical Commission (IEC), 3rd edition, 2000.

[25] N. Cho, C. Choi, and S. Lee, "Adaptive line enhancement by using an IIR lattice notch filter," IEEE Trans. Acoust., Speech, Signal Process., vol. 37, pp. 585-589, 1989.

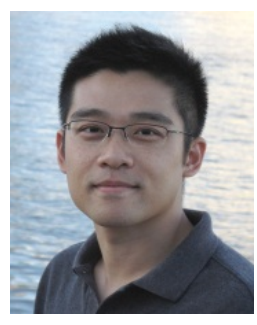

Jeffery C. Chan (S'12) is currently pursuing the $\mathrm{Ph} . \mathrm{D}$. degree in the School of Information Technology and Electrical Engineering, the University of Queensland, Australia. He obtained his B. Eng. (Hons.) degree in Mechatronic Engineering and M.Phil. degree in Manufacturing Engineering and Engineering Management from City University of Hong Kong in 2006 and 2009 respectively. Before joining the University of Queensland, he worked for a number of projects on machine fault diagnosis and energy conservation for 3 years. He is a member of IEEE. His research interests include signal processing, partial discharge, and condition monitoring of power systems.

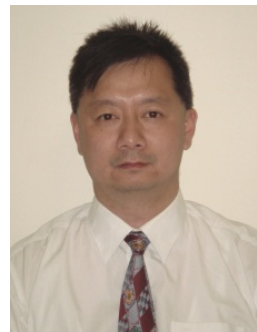

Hui Ma (M'95) received his B.Eng and M.Eng degrees from Xi'an Jiaotong University, China in 1991 and 1994, M.Eng (by research) degree from Nanyang Technological University, Singapore in 1998, and $\mathrm{PhD}$ degree from the University of Adelaide, Adelaide, Australia in 2008. Currently Dr. $\mathrm{Ma}$ is a research fellow in the School of Information Technology and Electrical Engineering, the University of Queensland, Australia. Prior to joining the University of Queensland, Dr. Ma has many years research and development experience. From 1994 to 1995, he was a researcher in Xi' an Jiaotong University, China. From 1997 to 1999, he worked as a firmware development engineer in CET Technologies Pte. Ltd., Singapore. He was with Singapore Institute of Manufacturing Technology as a research engineer from 1999 to 2003. His research interests include industrial informatics, condition monitoring and diagnosis, power systems, wireless sensor networks, and sensor signal processing.

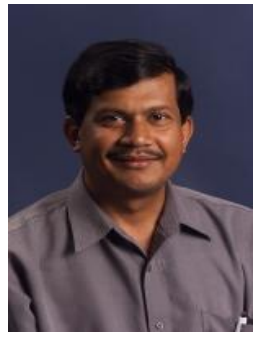

Tapan Kumar Saha (M'93, SM'97) was born in Bangladesh in 1959 and immigrated to Australia in 1989. He received his B.Sc Engineering (electrical and electronic) in 1982 from the Bangladesh University of Engineering \& Technology, Dhaka, Bangladesh, M. Tech (electrical engineering) in 1985 from the Indian Institute of Technology, New Delhi, India and $\mathrm{PhD}$ in 1994 from the University of Queensland, Brisbane, Australia. Tapan is currently Professor of Electrical Engineering in the School of Information Technology and Electrical Engineering, University of Queensland, Australia. Previously he has had visiting appointments for a semester at both the Royal Institute of Technology (KTH), Stockholm, Sweden and at the University of Newcastle (Australia). He is a Fellow of the Institution of Engineers, Australia. His research interests include condition monitoring of electrical plants, power systems and power quality.

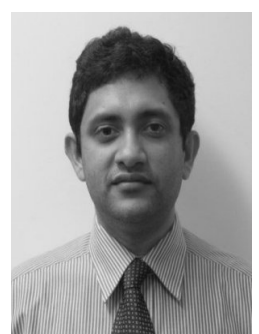

Chandima Ekanayake (M'00) received his B.Sc.Eng.(Hons) in 1999 from University of Peradeniya Sri Lanka. He obtained his Tech. Lic. and $\mathrm{PhD}$ from Chalmers University of Technology Sweden in 2003 and 2006 respectively. Currently he is a lecturer in the School of Information Technology and Electrical Engineering, the University of Queensland (UQ), Brisbane, Australia. Before joining UQ he was with University of Peradeniya Sri Lanka as a Senior lecturer. During his $\mathrm{PhD}$ studies he was working for a European Union Project called REDIATOOL where he engaged in research related to Diagnostics of Transformer Insulation from dielectric response measurements. From 2001, he has been involving on condition monitoring of transformers installed at Ceylon Electricity Board, Sri Lanka. He was the Chair of IEEE Sri Lanka Section in year 2006 and 2007. His research interests are condition monitoring of power apparatus, alternatives for insulating oil, transient studies on power systems and energy related studies. 